\title{
SiPM Use in Future Space Instruments
}

\author{
Jason Link ${ }^{* 11,2}$, T. Brandt ${ }^{1}$, G. De Nolfo ${ }^{1}$, J. Du Monthier ${ }^{1}$, J. Krizmanic ${ }^{1,2}$, J. \\ Mitchell $^{1}$, S. Mitchell ${ }^{1,3}$, K. Sakai ${ }^{1,2}$, M. Sasaki ${ }^{1,3}$, G. Suarez $^{1}$, M. Wiedenbeck ${ }^{4}$ \\ 1 NASA Goddard Space Flight Center, Greenbelt Maryland, 20720, USA \\ 2 CRESST-UMBC, Baltimore, Maryland 21250, USA \\ 3 CRESST-UMCP, College Park, Maryland 20742, USA \\ 4 NASA Jet Propulsion Laboratory, Pasadena, California 91109, USA
}

\begin{abstract}
Silicon photomultipliers (SiPM) are small solid state photodetectors which can provide single photon counting with high quantum efficiency, fast response and good charge resolution. These devices have similar gains $\left(10^{6}\right)$ as photomultiplier tubes but operate with a much lower bias ( $<60 \mathrm{~V})$. Many experiments are looking to use these devices instead of PMTs and ground-based experiments such as CMS, MAGIC and CTA are studying their use. There are also plans to utilize SiPMs in future astroparticle physics experiments like HELIX and TIGERISS for which they are particularly suited due to their compact size and low power consumption. In this paper we review the characteristics of SiPM and discuss the particular challenges using these in space experiments. We will report testing done in a $\mathrm{Pb}$ beam at CERN in November 2016 which included a SensL C-series SIPM of $6 \mathrm{~mm}$ in size. This SiPM was tested in a light box with a piece scintillator and two 1 inch Hamamatsu R1924A PMT so a comparison of the response of the two photodetectors can be made. In addition we placed the SiPM directly in the lead beam for a period to test if exposure to heavy ions would cause degradation in its sensitivity.
\end{abstract}

35th International Cosmic Ray Conference - ICRC2017-

10-20 July, 2017

Bexco, Busan, Korea

\footnotetext{
*Speaker.

†Email: Jason.Link@nasa.gov
} 


\section{Introduction}

In the last decade, Silicon photomultipliers (SiPMs) have matured and now offer compact, lightweight alternative to Photomultiplier Tubes (PMTs). These sensors are being used in several experiments like the T2K neutrino experiment and the CMS HCAL, as well as being considered for use in imaging atmospheric Cherenkov telescopes like MAGIC and CTA. They are also being considered for use in several NASA space and high altitude balloon experiments like HELIX, ASCOT, TIGERISS and HNX.

A particular concern for several experiments is radiation exposure of these sensors. While there has been a number of studies of the radiation hardness of these sensors due to their potential accelerator use [1] [2] the effects of heavy nuclei is far less well known. In this paper we discuss a 2016 test the High Energy Cosmic Ray group at NASA Goddard performed at CERN which included exposing a SiPM directly to a lead beam. During this test we also looked at how the SiPM's resolution and dynamic range compared to a PMT over a broad charge range to explore if it would be an appropriate device to use on some future ultra-heavy cosmic ray experiments (HNX, TIGERISS) we are developing [3].

\section{SiPM Sensors}

SiPMs consist of an array of photodiode elements, all running in Geiger mode. In this mode the photodiode elements are biased several volts higher than their breakdown voltage. When a photon strikes one of these elements it is absorbed by the silicon creating an electron-hole pair. The bias across the silicon creates an electric field that sweeps this charge carrier towards the anode (hole) or cathode (electrons). By running the photodiode elements in Geiger mode the applied bias accelerates the charge carrier to the point where it can create secondary charge pairs through impact ionization. This provides the SiPM gain of around $10^{6}$ which is comparable with Photomultiplier Tubes (PMTs). These photodiode elements also include an integrated resistor to quench the avalanche after tens of nanoseconds. The elements are read out in parallel and the summed output signal is proportional to the total number of cells that trigger from a photon striking them. Figure 1. below shows a picture of a SiPM element, basic electronic diagram and sample response to a flashing LED.

SiPMs have several advantages over PMTs. In addition to their smaller size, SiPMs do not require a glass vacuum tube making them a more rugged sensor. SiPMs also are not affected by a magnetic field which is particularly advantageous when used in a strong magnetic environment such as in an accelerator beamline or near a magnetic spectrometer as well as on detectors exposed to a changing magnetic environment. Finally, the bias voltage needed by SiPMs is usually well under $100 \mathrm{~V}$ versus PMTs which typically require voltages of hundreds or even thousands of volts. SiPMs do have some drawbacks over PMTs. Their gain is strongly temperature dependant as it depends on the difference between the bias voltage and breakdown voltage, which in turn depends on temperature. Additionally, though there have been improvements in the past few years, crosstalk between SiPM photodiode elements can yield to excess noise and linearity distortions which limit the dynamic range. 

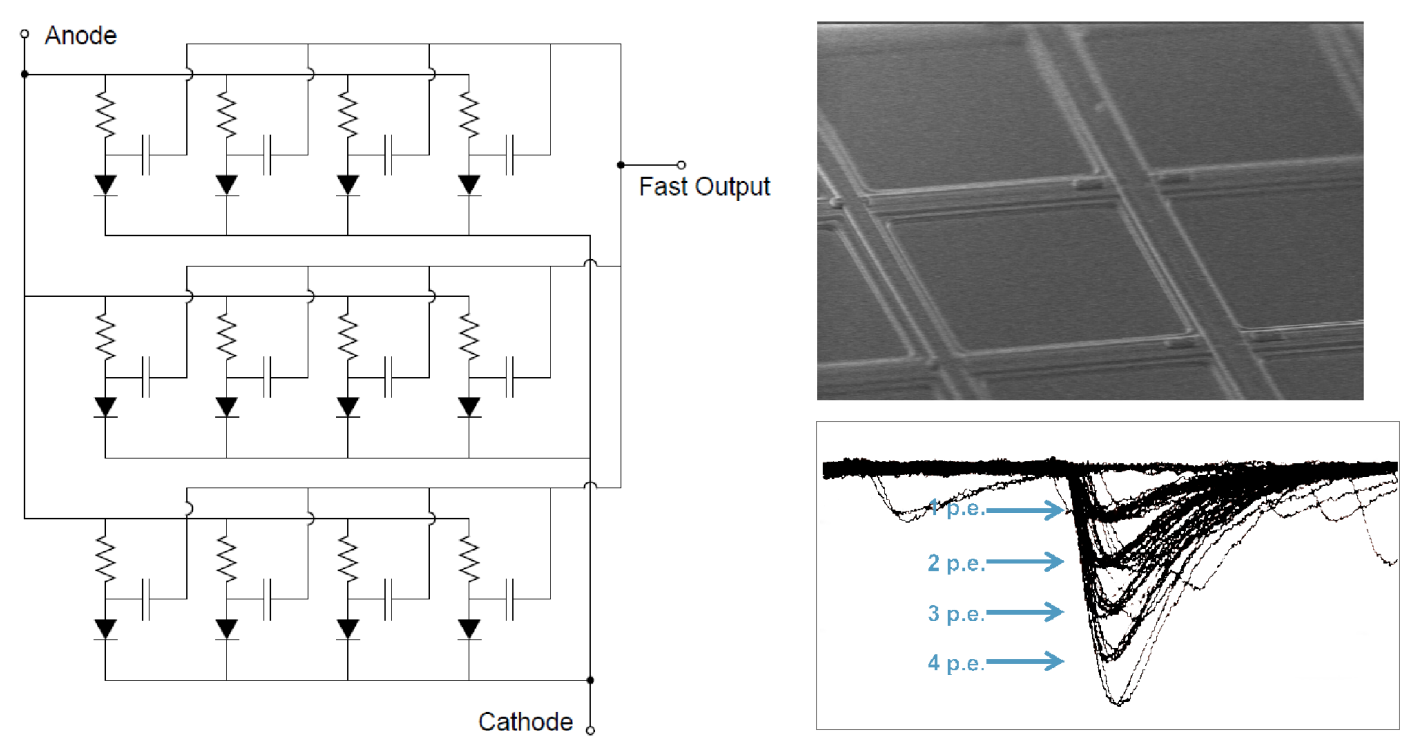

Figure 1: Left - Schematic diagram of a SIPM which consists of photodiodes plus quench resistors with a summed output. Upper Right - Image showing the microcell structure of the SiPM surface. Lower Right Oscilliscope traces showing the single PE sensistivity of a SiPM when illuminated by low-level light pulses. [4]

\section{Radiation Exposure and SiPMs}

Using SiPMs in space or near accelrator beams raises concerns about their operation and performance in the relavant radiation environments. Protons and neutrons may cause the sensor to have a degraded performance, latch up or become non-functional. Radiation damage studies of several SiPMs developed to work in the high-radiation environment of the CERN beamline have been performed by Musienko et al [1] for the CMS HCAL. They exposed these SiPMs to $62 \mathrm{MeV}$ protons at fluencies up to $1 \times 10^{12}$ protons $/ \mathrm{cm}^{2}$ at the UCL proton cyclotron. Significant increases of the dark current and noise were measured and one SiPM had a significant signal gain reduction which was found to be primarily due to a breakdown voltage shift measured after irradiation. The SiPMs did continue to function before and after being irradiated, the increase in dark current however means a higher noise threshold must be set reducing the sensitivity and dynamic range of the SiPM. For a typcial space environment the calculated fluence for an instrument launched in 2001 in a $590 \mathrm{~km}$ orbit with 29 degree inclination for 5 years is $2 \times 10^{10}$ protons $/ \mathrm{cm}^{2}$ [5].

A second concern is whether or not the sensor is affected when heavy atomic nuclei such as oxygen or iron directly strike it. Such an impact may damage the SiPM photodiode element with undesirable effects. If the element is rendered inert from the particle impact the SiPM would have a dead pixel and thus a loss of sensitivity. If the element is shorted such that it is always firing the SiPM then has an always on pixel that would lead to an increase in the baseline noise or dark current. While there have been studies done with the effects of particles such as protons and neutrons irradiating a SiPM we are unaware of any studies done on the effects of heavy atomic nuclei impacting a SiPM directly. 


\section{CERN 2016 Beamtest}

In November 2016 members of the Goddard High Energy Cosmic Ray group were granted time at CERN in the H8 North Area to test future detectors and sensors to measure ultra-heavy cosmic rays in a lead beam at various energies. Our primary motivation for this effort was to test the dynamic range and resolution of several silicon strip detectors. The results of this are discussed elsewhere in this conference [6]. We realized just before this test we had a opportunity to include a SiPM into our test setup and test their dynamic range and resolution. In addition we could expose a SiPM directly to a heavy ion beam and see if there was any effect on the SiPM response. The SiPM tests discussed in this paper occurred when the beam was tuned to an energy of $150 \mathrm{GeV} / \mathrm{n}$.

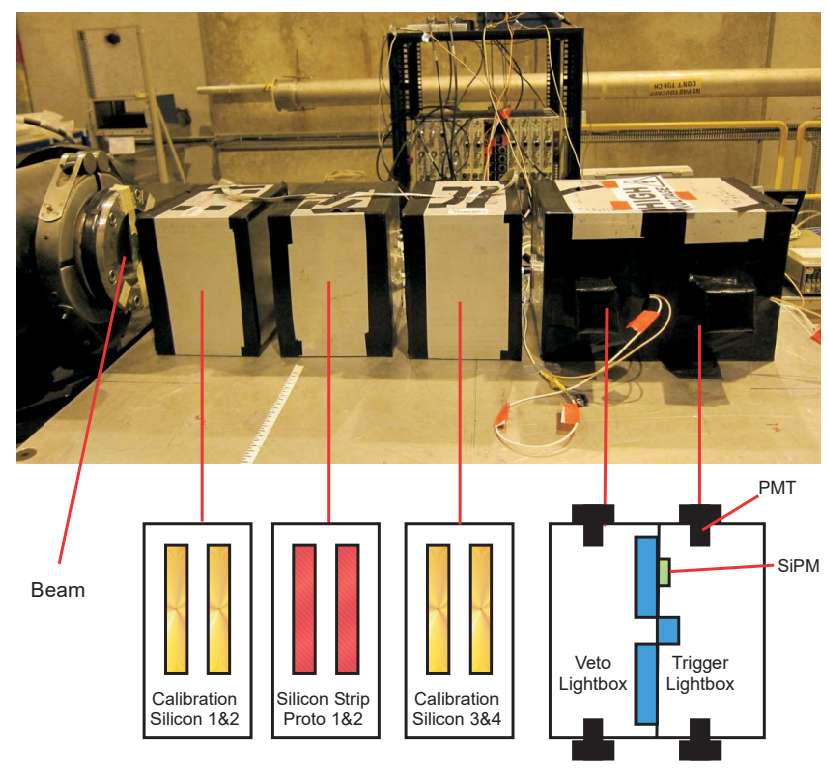

Figure 2: Detector Configruation for 2016 CERN Beam Test. The 3 boxes closest to the beamline exit contain silicon detectors. The last two boxes which are conjoined lightboxes that share a common wall contain PMTs and scintillator to form a trigger of the instrument readout. A SiPM was installed in the trigger lightbox.

Our test setup in the beam is shown in Figure 2. . We set our detectors as close to the beam as possible to minimize interactions in air between the beamline exit and our detectors. The calibration silicon enclosures each contain two 500 micron thick $10 \mathrm{~cm}$ x $10 \mathrm{~cm}$ PAD detectors. The silicon strip prototypes enclosure were manufactured by Micron Semiconductors LTD. Each contains two 500 micron thick silicon strip detectors with $3 \mathrm{~mm}$ pitch covering a $10 \mathrm{~cm}$ x $10 \mathrm{~cm}$ region. We configured our electronics to read out both the cathode and anode side of the detector. Behind the silicon detectors in the beamline are two joined boxes. These boxes are lined with Tyvek and each has scintillator inside with two R1924 PMTs looking in and form our readout system trigger. The veto lightbox has a square piece of scintillator along the far wall perpendicular to the beam with 1 inch circular hole cut out of the scintilator and centered on the beam. The trigger lightbox has a 1 inch diameter piece of scintillator centered on the beam. The event trigger is formed by accepting a concidence trigger from the two PMTs looking into the trigger lightbox while requiring thet two PMTs looking into the veto lightbox see no signal. 
We installed a SensL MicroFC-SMA-60035 into the trigger lightbox as shown in Figure 3. This is a $6 \mathrm{~mm}$ square SiPM mounted on an evaluation board with SMA connectors. This SiPM has a microcell size of 35 micron and a breakdown voltage between 24.2-24.7 V. [4] The sensor was biased at $28 \mathrm{~V}$ during our testing and read out through the same readout electronics as the PMTs installed in the veto and trigger lightboxes.

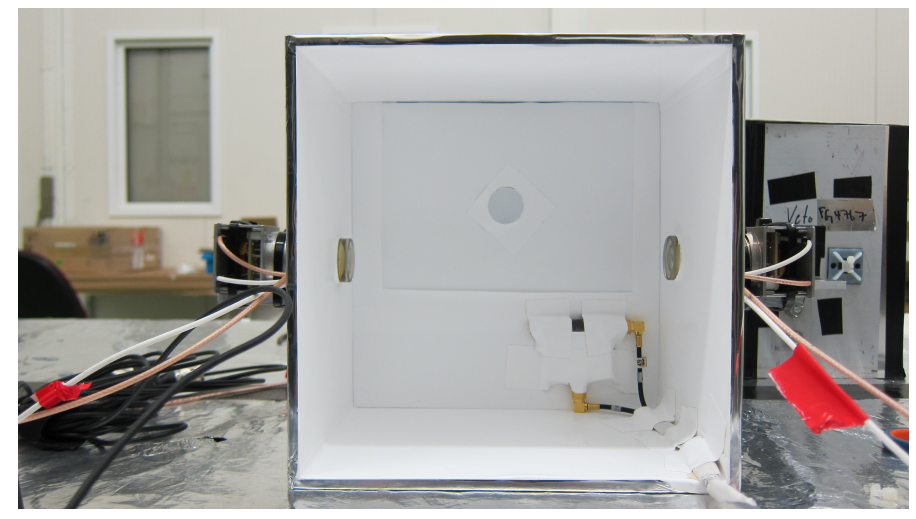

Figure 3: Internal view of the trigger lightox showing the small 1" scintillator disc that is near the center of the box, the two R1924A PMTs looking into the lightbox from the left and right side and the SiPM in the lower right of the box facing the camera.

\subsection{SiPM Dynamic Range and Resolution}

One of the motivations we had in including a SiPM in our test setup was to see how it compared to PMTs in terms of dynamic range and resolution over a broad charge range. We conducted runs in the beam with a lead primary beam as well as runs with a polyethelene target in the led primary beam and selecting different $\mathrm{A} / \mathrm{Z}$ fragments using the beam magnets.

In Figure 4 we show data from two of the pure lead beam runs. The charge gain conversion is done by making a linear fit between the PHA signal peak and assuming it is centered on a $\mathrm{Z}=82$ lead peak. We then use data from the silicon detectors to apply a charge correction to the data from the SiPM and PMT to improve resolution. We then fit a gaussian to the resulting data distribution. We see that the peak and sigma is very similar between the SiPM and PMT for both of the runs in Figure 4.

In Figure 5 we show data from the entire accelerator test which included runs where we had material in the beam to cause interactions to get a flux of particles lighter than lead. The PMT and SiPM have the linear charge gain fit and then correction from the silicon detector signal as described above. The peak shading is based on charge cuts from a multiple gausian fit to the silicon data. The resolution of the SiPM and PMTs does not allow this fitting to occur easily so we used the silicon to determine the shading. We see that the SiPM compares very favoribly to the PMT in terms of dynamic range and resolution. This result suggests that SiPMs may be able to replace PMTs in the proposed HNX CosmicTIGER detector as well as our TIGERISS concept. [6] We will be conducting further tests, in particular looking at effects of temperature variation on the SiPMs response, to verify this. 

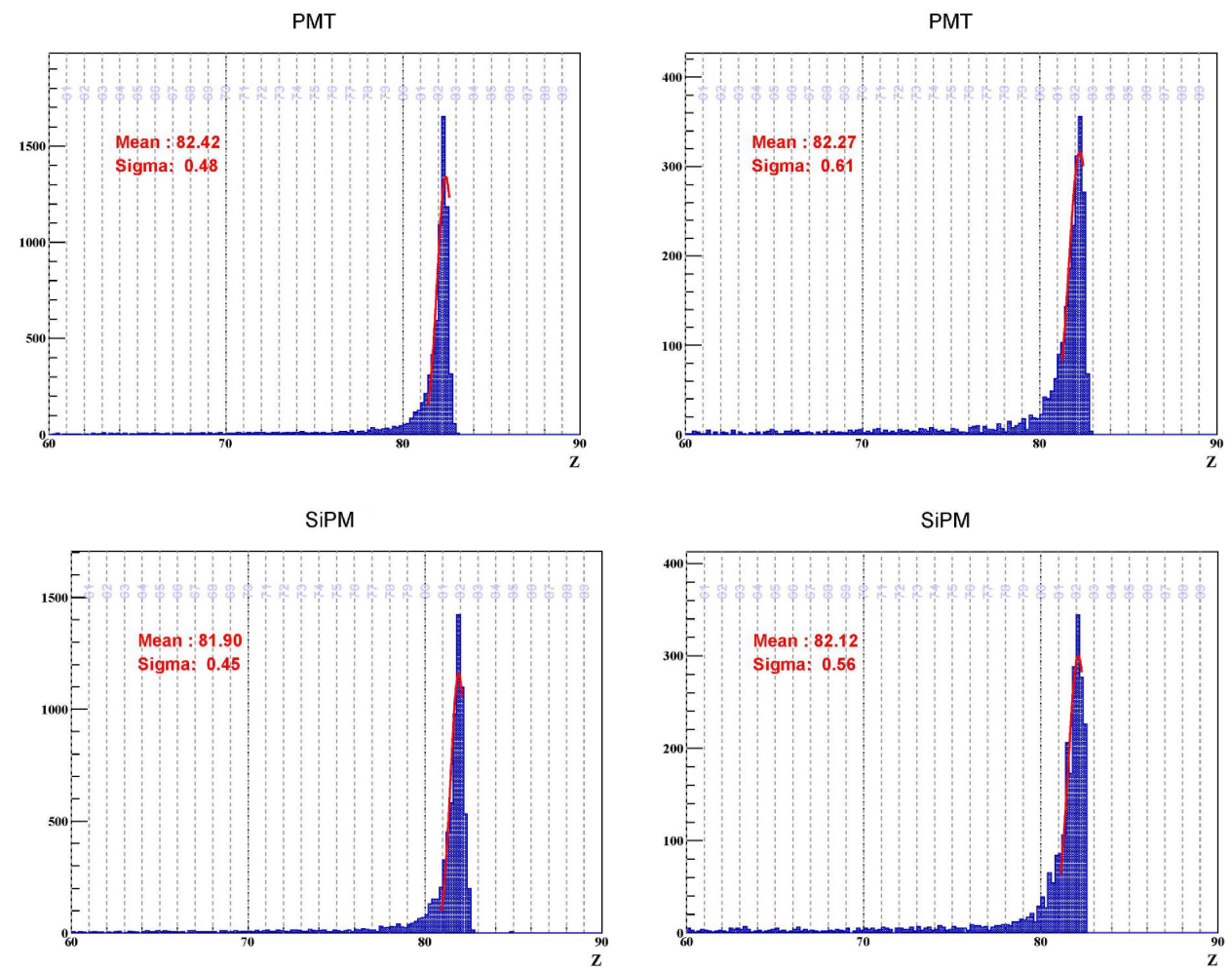

Figure 4: Plot of PMT and SiPM signal from $150 \mathrm{GeV} / \mathrm{n} \mathrm{Pb}$ beam passing through scintillator in Trigger box. The plots on the left are prior to the SiPM being exposed to the $\mathrm{Pb}$ Beam. the plots on the right are after the SiPM was exposed.

\subsection{Effects on SiPM direcly exposed to Lead Beam}

As mentioned earlier, we are not aware of any tests where SiPMs have been exposed directly to heavy ions. If heavy ions cause damage to the pixels when they pass through the sensor this could be a detriment for using these sensors for astrophysical applications on balloon and satellites. We unfortunately had far less time to conduct a SiPM exposure test than we would have liked due to a variety of factors including unexpected down time of the beam, some readout electronics issues and a lower beam fluence than we hoped while characterizing our silicon detectors. In the limited time we had available we took data in a $150 \mathrm{GeV} / \mathrm{n}$ lead beam for 19 minutes with $\mathrm{A} / 2=2$ with our setup as show in Figure 2. We then moved the conjoined veto and trigger boxes so the lead beam passed directly through the SiPM and exposed it in the Pb beam for 13 minutes. We then moved the conjoined veto and trigger boxes to their original configuration and took data for 25 minutes. We had two significant issues during the test. First, we did not have time to modify our readout and enable it to trigger and readout the silicon and SiPM while the SiPM was directly in the beam. We were able to run a cable from the SiPM output to an oscilloscope and see that the SiPM was clearly being hit by heavy particles but could not record any data during this time. Second the beam fluence whilte the SiPM was being directly exposed decreased significantly. We saw this immediately from monitoring the SiPM on the oscilloscope as well as the beam status report. We were able to record the time of the change in fluence but it meant we got less lead exposure than 

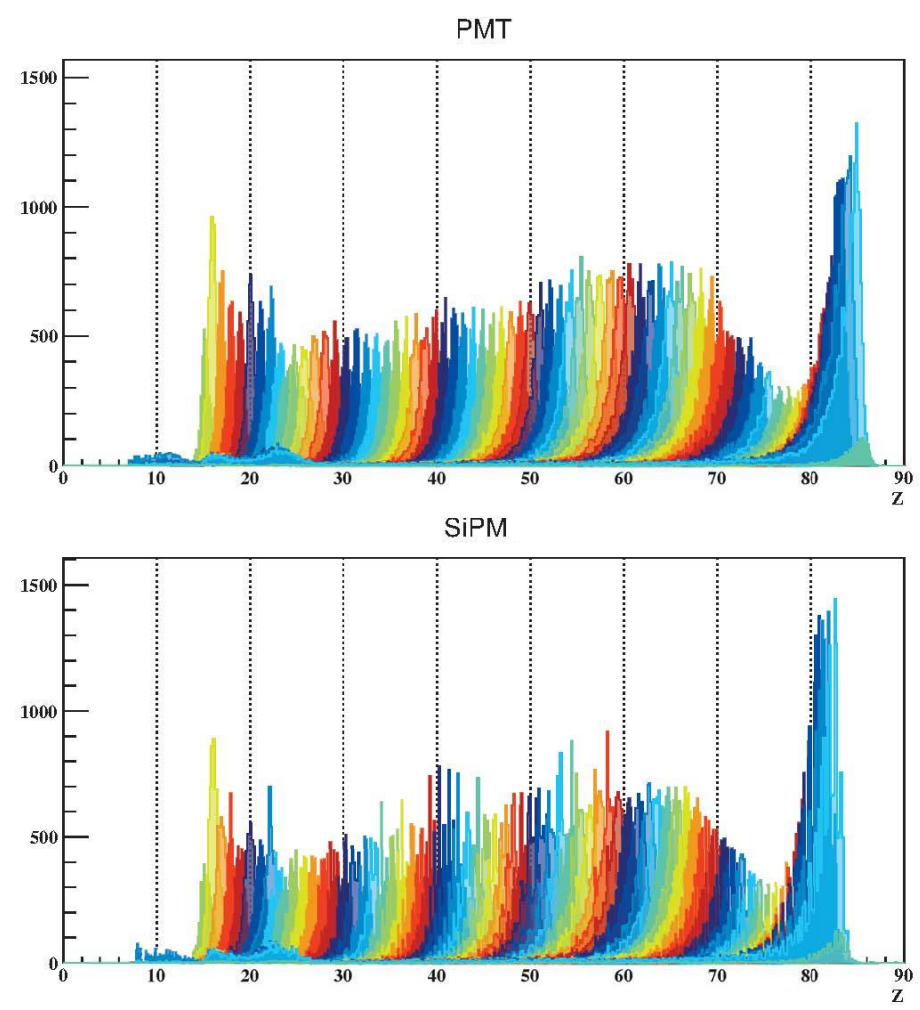

Figure 5: Plot of PMT and SiPM signal based on all events collected during the beam run. This includes run where we put material in the beam to generate lighter elemets. The dynamic range and resolution of the SiPM compares very favoribly to the PMT.

we hoped.

Based on the number of events we collected before and after the exposure test and the time we recorded the beam fluence changes we calculate a lower exposure limit of 6426 events striking the SiPM during its 15 minute exposure. This calculation is a lower limit as we do not account for any events lost due to the deadtime in the readout system. Figure 4 shows data from the beam runs before and after the SiPM was exposed to the lead beam. We note that the resolution of both the SiPM and PMT are a bit worse after exposure but this is likely due to the change in the beam rather than anything with the sensors. This is a promising initial result.

\section{Conclusion}

We used a SiPM to look at events in a $\mathrm{Pb}$ beam in 2016 and found that its dynamic range and resolution compared favoribly to a PMT. Based on this performance we are planning to conduct further tests in November 2017 when we have 2 weeks of time at CERN in a Xenon ( $Z=54)$ beam. For this run we plan to build a dedicated lightbox for the SensL SiPM and possibly test an SiPM from Hammamatsu and other manufactureers. The dedicated lightbox will enable us to collect SiPM data when it is in the beam. In addition it will enable us to do longer exposure tests without disturbing the trigger setup for the silicon detectors. 


\section{References}

[1] Musienko et al., Radiation Damage Studies of Silicon Photomultipliers for the CMS HCAL Phase 1 Upgrade, Nucl. Inst. Meth. Phys. Res. A 787, 319-322 (2015).

[2] Qjang, Y et al., Radiation Hardness Test of SiPMs for the JLab Hall D Barrel calorimeter, Nucl. Inst. Meth. Phys. Res. A 698, 234 (2013).

[3] Mitchell, J.W. et al., Ultra-Heavy GCR measurements beyond SuperTIGER: HNX and TIGERISS, Proc. of 35rd ICRC Conference, Bursan, South Korea (2017).

[4] SensL C-series Datasheet: http://sensl.com/downloads/ds/DS-MicroCseries.pdf

[5] Poivey, C., Radiation Hardness Assurance for Space Systems, Part IV of the short Course presented at the 2002 Nuclear and space Radiation Effects Conference, Phoenix Az, (15 July 2002).

[6] Krizmanic, J.F. et al., HNX/TIGER ISS Silicon Strip Detector Response to Nuclei Measured in a Lead Test Beam, Proc. of 35rd ICRC Conference, Bursan, South Korea (2017). 